\title{
Establishment of a non-coding RNAomics screening platform for the regulation of $K R A S$ in pancreatic cancer by RNA sequencing
}

\author{
LI ZHANG, SHUANGNI YU, CUIPING WANG, CONGWEI JIA, ZHAOHUI LU and JIE CHEN \\ Department of Pathology, Peking Union Medical College Hospital, Chinese Academy of Medical Sciences \\ and Peking Union Medical College, Beijing 100730, P.R. China
}

Received April 18, 2018; Accepted August 9, 2018

DOI: $10.3892 /$ ijo.2018.4560

\begin{abstract}
KRAS oncogene point mutations occur in $>95 \%$ of patients with pancreatic cancer. The KRAS protein can activate various downstream effector molecules that affect proliferation and differentiation. MS2 binding sites (MS2bs) are RNAs of $19 \mathrm{bp}$ in length that can bind MS2 coat proteins with their specific stem-loop structure. The MS2 binding site sequence of the 19-nucleotide stem-loop is ACATGAGGATCACCCATGT. We constructed an expression vector that expresses the KRAS non-coding region coupled with 12 copies of MS2bs in series and established a high-throughput library for collecting microRNA (miRNA or miR)- and long non-coding RNA (lncRNA)-omics that regulate KRAS. To the best of our knowledge, this is the first study to combine RNA-protein interactions with RNA sequencing to obtain $K R A S$-associated non-coding RNAs. As a result, we identified several miRNA precursors that belong to the let-7 and miR-34, -30 and -143 families, as well as relevant lncRNAs and their families (MALAT1, MEG3_2 and TUG1_1-4). Our databank of non-coding RNAs (mainly miRNAs and lncRNAs) that regulate $K R A S$ is expected to greatly enhance our understanding of $K R A S$ regulation-associated tumorigenesis and may aid in the development of gene therapies for pancreatic cancer.
\end{abstract}

\section{Introduction}

Pancreatic cancer (PC) is a highly malignant tumor that is characterized by early metastasis. In Western countries, PC is one of the leading causes of cancer-related mortality (1). Researchers have found that KRAS oncogene point mutations occur in $>95 \%$ of patients with PC (2). Genetic alterations of oncogenes, such as KRAS and HER-2, and tumor suppressor

Correspondence to: Dr Jie Chen, Department of Pathology, Peking Union Medical College Hospital, Chinese Academy of Medical Sciences and Peking Union Medical College, 1 Shuaifuyuan, Wangfujing, Dongcheng, Beijing 100730, P.R. China

E-mail:xhblk@163.com

Key words: pancreatic cancer, KRAS, microRNA, long non-coding RNA, next-generation sequencing genes, such as $p 53, p 16$ and SMAD4, are the main molecular alterations associated with the occurrence and development of PC (3). Among these, KRAS oncogene point mutations occur in the early stages of tumor development, as well as throughout the growth stages. Oncogenic KRAS promotes pancreatic tumorigenesis through the activation of multiple downstream pathways, including phosphatidylinositol-3-kinase, extracellular signal-regulated kinase and nuclear factor- $\kappa B$. Since these molecules significantly affect cell proliferation and differentiation, the timely and effective treatment of PC through the inhibition of KRAS in the early stages of tumor development is critical.

MicroRNAs (miRNAs or miRs) are small non-coding RNAs consisting of 20-22 nucleotides (nt) (4) that play a key role in tumor development and progression (5). miRNAs have been found to be differentially expressed at various stages of PC progression (6). miRNAs have been previously found to play a key role in the regulation of $K R A S$. In particular, miR-193b was verified as a direct inhibitor of $K R A S$, resulting in the modulation of Akt and extracellular signal-regulated kinase pathways and in the suppression of pancreatic ductal adenocarcinoma (PDAC) cell growth (7). miR-206 has also been found to directly target KRAS and to function as a tumor suppressor in PDAC cells by blocking cell cycle progression, proliferation, migration and invasion (8), while miR-143 expression has been found to significantly decrease KRAS mRNA and protein levels (9). KRAS has also been identified as a potential direct target of miR-217, which acts as a tumor suppressor in PDAC cells (10). Furthermore, miR-96 has been shown to regulate tumorigenesis through the inhibition of cell proliferative and invasive activities by targeting KRAS (11).

Long non-coding RNAs (lncRNAs) are an important class of non-coding RNAs $>200 \mathrm{nt}$ in length (12), that rarely participate in protein coding, but are involved in epigenetic and transcriptional regulation, as well as in various other levels (13). Recent studies have found that IncRNAs play a central role in the regulation of a wide range of biological processes $(14,15)$ and are closely associated with a number of human diseases, including several types of cancer (16-18). Furthermore, several differentially expressed lncRNAs have been identified in primary and metastatic PC (19); thus, lncRNAs may be associated with PC metastasis. Kim et al demonstrated that HOTAIR functions as an oncogene in $\mathrm{PC}$ and is associated with a poor prognosis (20). A previous 
microarray analysis revealed that HOTTIP was one of the most significantly upregulated lncRNAs in PDAC tissues compared with adjacent normal pancreatic tissues (21), whereas lncRNAs MALAT1 and HULC have been shown to be associated with a poor prognosis in PC (22-24). However, there is currently little information on the association between IncRNAs and KRAS, at least to the best of our knowledge.

MS2 capsid (coat) protein is the envelope protein found on bacteriophage MS2 that specifically interacts with MS2 binding sites (MS2bs), which are RNA stem-loop structures composed of 19 base pairs (25). MS2 protein-MS2bs RNA binding has been used to study intra- and extracellular RNA-protein interactions (26). Some researchers have even made 12 MS2bs binding sites in series (27). Zhou et al used MS2-MBP to purify RNA-protein complexes in vitro (28). A pMAL protein expression vector system was previously used to induce the expression of a target protein. MBP fusion proteins can enhance the solubility of target proteins, and MBP can bind with resins. Corcoran et al and others have utilized an MS2-maltose binding protein (MBP) fusion protein and resin for affinity chromatography analysis of RNA-protein complexes and the identification of binding proteins $(29,30)$. Bardwell et al used MS2 capsid protein and MS2bs structure to purify RNA-protein complexes (31). SenGupta et al established an MS2 capsid protein-based yeast RNA 3-hybrid system, which can identify RNA-protein interactions (32).

The importance of the target genes regulated by non-coding RNA molecules reflects the importance of these small RNA molecules. Since KRAS mutations are one of the most significant genetic alterations in PC, the identification of non-coding RNAs associated with KRAS regulation is crucial to the study of the pathogenesis of PC. In this study, we constructed an expression vector that expresses KRAS non-coding region coupled with 12 copies of MS2bs in series, and established a highthroughput library for collecting miRNA- and lncRNA-omics that regulate KRAS. To the best of our knowledge, this is the first study to combine RNA-protein interactions with RNA sequencing to obtain $K R A S$-associated non-coding RNAs. We successfully constructed the pcDNA3.1-KRAS-12xMS2bs plasmid, and used pMAL-c5x-MS2 vectors to induce the expression of MS2-MBP fusion protein. Subsequently, RNA that was associated with KRAS was obtained through affinity chromatography resin experiments. Finally, the miRNA and lncRNA data of interest were obtained through next-generation RNA sequencing. We identified a number of miRNA precursors that belong to the let-7 and miR-34, -30 and -143 families, as well as relevant lncRNAs and their families (MALAT1, MEG3_2 and TUG1_1-4). Our databank of non-coding RNAs (mainly miRNAs and lncRNAs) that regulate KRAS is expected to greatly enhance our understanding of KRAS regulation-associated tumorigenesis and may thus aid in the design of novel gene therapies for PC.

\section{Materials and methods}

pcDNA3.1-KRAS-12xMS2bs plasmid vector construction. The MS2 binding site sequence of the 19-nt stem-loop was ACATGAGGATCACCCATGT. The sequences were sythesized by Beijing PolePolar Biotechnology Co., Ltd. (Beijing, China). The underlined letters in the sequences indicate MS2 binding sites (MS2bs), which are RNA stem-loop structures composed of 19 base pairs (26). First, the following sequences were synthesized: 12xMS2bs-A, 5'-AGCTTGGTACCGAGC TCGGATCCACATGAGGATCACCCATGTACATGAGGAT CACCCATGTGAGACCATCCACTAGTCTCGAGTCTAGA GGGCCCGT-3'; 12 x MS2bs-B,5'-TAAGCGTCTCACATGAG GATCACCCATGTACATGAGGATCACCCATGTGAGACCC TAGAAGCTCGAGTTA-3'; 12xMS2bs-C, 5'-TAAGCGTCTC CCATGTACATGAGGATCACCCATGTACATGAGGATCAC CCATGTCTCGAGTCTAGAGGGCCCGTTTAAACCCGC-3'. The synthesized sequences were then cloned into a $\mathrm{T}$ vector (Promega, Madison, WI, USA) lacking the BsaI, BsmBI and XhoI restriction sites and sequenced. 12xMS2bs-B was digested with $B s m B I$ and $X h o I$ and the fragment was recovered. The BsaI/XhoI-digested 12xMS2bs-B fragments were ligated to $12 \mathrm{xMS} 2 \mathrm{bs}-\mathrm{A}$, which was digested with $\mathrm{Bs} a \mathrm{I}$ and $\mathrm{XhoI}$ and then sequenced to obtain $12 \mathrm{xMS} 2 \mathrm{bs}-\mathrm{A} 4$; another $\mathrm{BsaI} / \mathrm{XhoI}$ digested the $12 \mathrm{xMS} 2 \mathrm{bs}-\mathrm{B}$ vector to obtain $12 \mathrm{xMS} 2 \mathrm{bs}-\mathrm{B} 4$ and the $12 \mathrm{xMS} 2 \mathrm{bs}-\mathrm{A} 4$ vector to obtain $12 \mathrm{xMS} 2 \mathrm{bs}-\mathrm{A} 6$. The $12 \mathrm{xMS} 2 \mathrm{bs}-\mathrm{C}$ was ligated to $12 \mathrm{xMS} 2 \mathrm{bs}-\mathrm{B} 4$ and sequenced to obtain 12 x MS2bs-C6, which was ligated to $12 \times$ MS2bs-A6 to produce $12 \mathrm{xMS} 2 \mathrm{bs}-\mathrm{T}$. Then, $12 \mathrm{xMS} 2 \mathrm{bs}-\mathrm{T}$ was digested with BamHI and XhoI to obtain the 12xMS2bs fragment, which was connected to the pcDNA3.1(+) plasmid (Invitrogen/Thermo Fisher Scientific, Waltham, MA USA) to obtain the final sequence. The $12 \mathrm{xMS} 2 \mathrm{bs}-\mathrm{C} 6$ fragment was also ligated to the $12 \mathrm{xMS} 2 \mathrm{bs}-\mathrm{B} 4$ vector and sequenced to produce $12 \mathrm{xMS} 2 \mathrm{bs}-$ $\mathrm{C} 10$ for future use. The untreated group was not treated with any transfection reagents and no transfection plasmid are added. NC was the negative control, a meaningless control of sequence disruption, and the pcDNA3.1 vector was used here. MOCK was the blank control, and only transfection reagents were added, and no transfection plasmid was added.

3'-UTR1-KRAS-12xMS2bs, 3'-UTR2-KRAS-12xMS2bs, 3'-UTR3-KRAS-12xMS2bs, and 3'-UTR4-KRAS-12xMS2bs construction. The KRAS 3'-UTR is very long; thus, to make it more readily transfectable into PC cells in order to successfully express RNA, the KRAS 3'-UTR was divided into 4 sections. Subsequently, the abovementioned $12 x M S 2 b s$ sequences were cloned into a $\mathrm{T}$ vector and sequenced. To obtain the 10xMS2bs fragment, 12xMS2bs-C10 was digested with BsmBI and XhoI, and then connected to plasmid B mentioned above to produce Fragment II. Fragments I and II were amplified by polymerase chain reaction (PCR) and finally integrated together by PCR. The integrated fragment was then cloned into a $\mathrm{T}$ vector to obtain the desired target sequence. The target sequence was then connected to the pcDNA3.1 plasmid to obtain the desired recombinant plasmid. Human pancreatic adenocarcinoma cells (PANC-1) was obtained from ATCC (Manassas, VA, USA). The cells were cultured in appropriate medium (Mediatech, Manassas, VA, USA) containing $10 \%$ fetal bovine serum (Thermo Fisher Scientific) and maintained at $37^{\circ} \mathrm{C}$ and $5 \% \mathrm{CO}_{2}$.

MBP-MS2 fusion protein expression. The PMAL-c5xpMAL vector [New England Biolabs (NEB), Ipswich, MA, USA) was used for the expression and purification of fusion proteins. Using the strong promoter 'Ptac' and the MBP translation initiation signal, the target gene (MS2)-malE (encodes MBP protein) fusion protein was expressed. A small amount of 


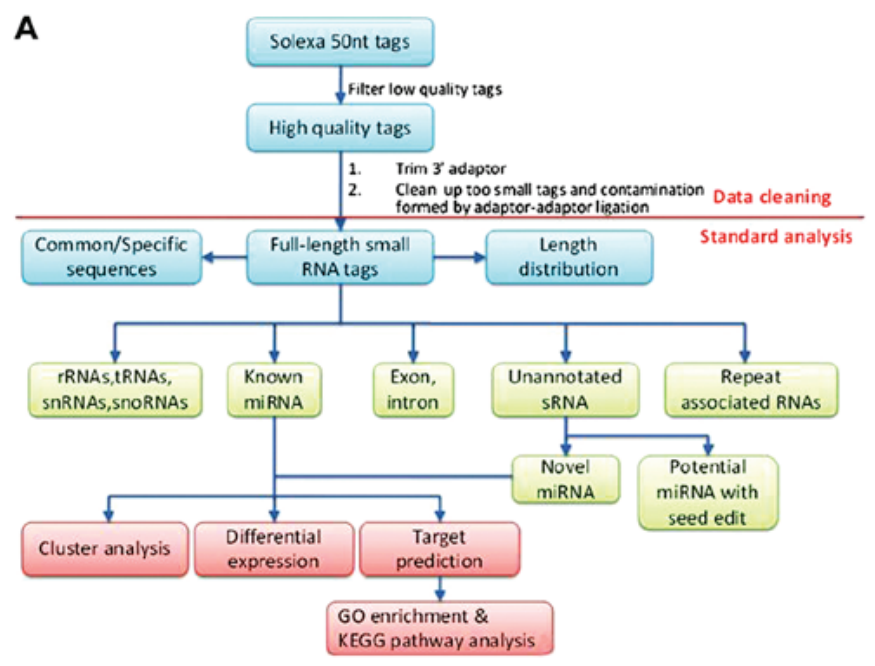

C

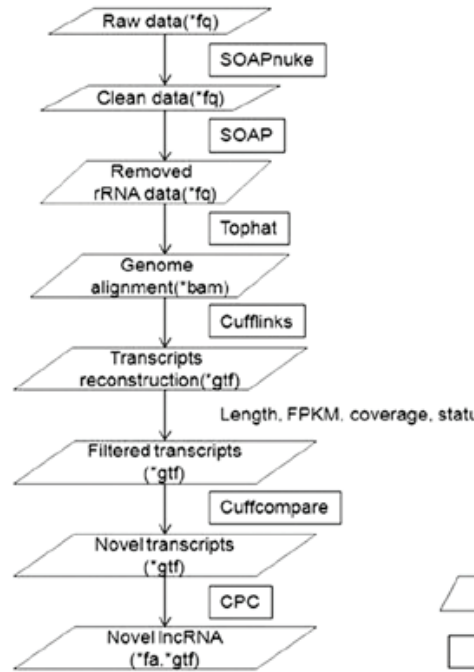

B

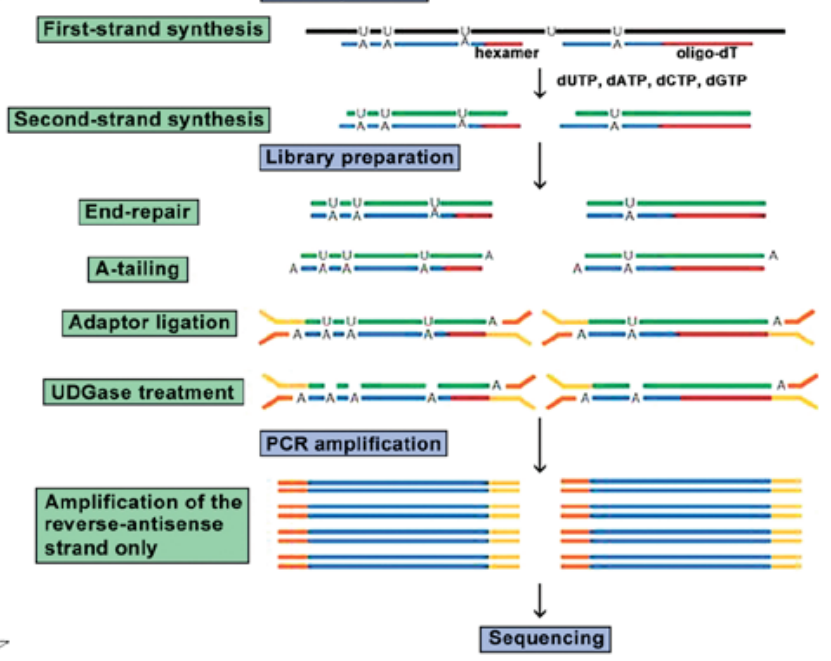

Figure 1. RNA sequencing procedures and bioinformatics analysis. (A) The small RNA sequencing procedure is depicted that was performed at the Beijing Genomics Institute. (B) Representative image of the long non-coding RNA (lncRNA) sequencing procedure. (C) Representative image of the lncRNA bioinformatics analysis of single-sample predictions.

the recombinant pMAL-c5x plasmid was transformed into Escherichia coli DE3 (Tiangen Biotech, Beijing, China), coated on plates and incubated overnight. We selected and collected a single bacteria which was gently agitated in vitro. The bacteria was then vigorously shaken. When the optical density (OD) value was 0.6 , isopropyl- $\beta$-D-1-thiogalactopyranoside (IPTG) was added to induce fusion protein expression. The bacterial cells were collected by centrifugation $\left(15,000 \times \mathrm{g}, 4^{\circ} \mathrm{C}\right)$ and disrupted with lysis buffer, then centrifuged $\left(15,000 \times \mathrm{g}, 4^{\circ} \mathrm{C}\right)$ again. The supernatant was collected for further purification with linear starch and finally rinsed with maltose-containing column eluent to obtain the MBP-MS2 fusion protein.

Affinity chromatography. We transfected the KRAS-12xMS2bs plasmid into the PANC-1 cells using Lipofectamine 2000 reagent (Thermo Fisher Scientific) and total RNA was extracted after 48 h. The column (\#732-6008; Bio-Rad, Hercules, CA, USA) was infused with resin and washed several times with washing buffer. Subsequently, KRAS-12xMS2bs RNA and the MBP-MS2 fusion protein were added to the column followed by incubation on ice for $30 \mathrm{~min}$. The column was then washed with eluent containing maltose (Bioroyee Biotech, Beijing,
China) and KRAS-12xMS2bs-MS2-MBP complexes were obtained. Finally, RNA was separated with chloroform and isopropanol to successfully obtain $K R A S$-associated RNA.

Small RNA sequencing. All sequencing procedures were performed at the Beijing Genomics Institute (Beijing, China). Following the removal of adaptor sequences, low-quality tags and contaminants, clean reads were used for bioinformatics analysis. The small RNA tags were mapped to the human genome to analyze their expression and distribution. Subsequently, we screened against the Rfam 10.1 and GenBank databases to remove the fragments of ribosomal RNA (rRNA), transfer RNA (tRNA), small nuclear RNA (snRNA) and small nucleolar RNA (snoRNA). After eliminating repeat-associated small RNA (sRNA), the degradation fragments of mRNA and identifying conserved miRNAs, the reads that did not match the above-mentioned databases were predicted using MIREAP (BGI, Beijing Genomics Institute, Beijing, China) (Fig. 1A).

lncRNA sequencing. After extracting total RNA from the samples, mRNA and non-coding RNA were enriched by removing the rRNA with a kit (BGI, Beijing Genomics Institute); 
mRNA could also be removed by removing poly-A RNAs, since lncRNAs also have a poly-A tail. Using fragmentation buffer (BGI, Beijing Genomics Institute), mRNA and non-coding RNA were fragmented into short fragments ( 200-500 nt), then first-strand cDNA was synthesized by random hexamer primers using the fragments as templates, and dTTP was substituted by dUTP during the synthesis of the second strand. Short fragments were purified and resolved with Elution Buffer (BGI, Beijing Genomics Institute) for end reparation and single adenine addition. Subsequently, the short fragments were connected with adapters and the second strand degraded using uracil-N-glycosylase. Following agarose gel electrophoresis, suitable fragments were selected for PCR amplification as templates. During the quality control (QC) steps, the Agilent 2100 Bioanalyzer (Agilent Technologies, Santa Clara, CA, USA) and ABI StepOnePlus Real-Time PCR System (Applied Biosystems/Thermo Fisher Scientific) were used for the quantification and qualification of the sample library. Finally, the library was sequenced using an Illumina HiSeq ${ }^{\mathrm{TM}} 2000$ or another sequencer when necessary (Fig. 1B). All sequencing procedures were performed at the Beijing Genomics Institute.

Novel miRNA prediction. miRNA hairpins are mostly located in intergenic regions, introns, or reverse repeat sequences of exons. The characteristic hairpin structure of miRNA precursors may be used to predict novel miRNAs. MIREAP prediction software (http://sourceforge.net/projects/mireap/) was used to predict novel miRNAs by exploring secondary structure, the Dicer cleavage site and the minimum free energy of the unannotated small RNA tags that could be mapped to the genome. Key search and selection conditions were as follows: i) The tags used to predict novel miRNA were from unannotated tags, which match the reference genome, align to intronic regions, or align to antisense exonic regions; ii) sequences and structures of hairpin miRNA able to fold into appropriate secondary structures and containing mature miRNA in one arm of the hairpin precursors were considered as candidate miRNA genes; iii) both the mature miRNA strand and its complement had 2-nt 3'-overhangs; iv) hairpin precursors lacking large internal loops or bulges; v) stable hairpin secondary structures with a free energy of hybridization $\leq-18 \mathrm{kcal} / \mathrm{mol}$; and vi) alignment results showed at least 5 mature miRNAs with predicted hairpins.

IncRNA bioinformatics analysis of single-sample predictions. Primary sequencing data produced by Illumina Hiseq ${ }^{\mathrm{TM}} 2000$, referred to as 'raw reads', which were filtered into 'clean reads' by removing adaptors, contained and low-quality reads. Due to unstable rRNA removal efficiency, it was also necessary to remove the rRNA-containing reads by alignment. We utilized reference annotation-based assembly to reconstruct transcripts, while background noise was reduced using fragments per kilobase of transcript per million mapped reads (FPKM) and coverage threshold. Novel transcripts were detected through comparison with a reference, and the coding potential of these transcripts was then calculated to identify the novel lncRNA. Since lncRNAs may be processed to yield small RNAs, small RNA precursor prediction was also performed. lncRNAs were also classified by family, RNA structure and sequence similarities, all of which helped reveal the potential function of the lncRNAs (Fig. 1C).
Detecting non-coding RNA molecule effects. We introduced exogenous miRNA molecules to PC cells and then isolated RNA to detect the effect (inhibition/activation) of the non-coding RNA molecules acting on KRAS sequences by reverse transcription-quantitative PCR (RT-qPCR) and western blot analyses. miRNA mimics of highly expressed small fragments matching several miRNA precursors (hsalet-7a, hsa-let-7i and hsa-miR-30b) were synthesized (Shanghai GenePharma Co., Ltd., Shanghai, China) and transfected into the PC cells. For quantitative PCR, total RNA was isolated from the PC cells using TRIzol reagent (Invitrogen/Thermo Fisher Scientific), according to the manufacturer's instructions. Quantitative PCR assay was performed with SYBR-Green PCR Master Mix using a StepOnePlus Detection System (Applied Biosystems/Thermo Fisher Scientific) to detect the KRAS mRNA expression levels. The KRAS primers used in this study are listed as follows: forward, 5'-GACTCTGAAGAT GTACCTATGGTCCTA-3' and reverse, 5'-CATCATCAACA CCCTGTCTTGTC-3'. For the reaction system, a 96-well plate was used, and each sample was set to 3 replicate wells with a total volume of $20 \mu \mathrm{l}$ per well. After the addition was completed, the samples were centrifuged at $2^{\circ} \mathrm{C}, 800 \mathrm{x} g$ for $2 \mathrm{~min}$. The reaction procedure was carried out on a StepOnePlus Real Time PCR System instrument (Applied Biosystems/ Thermo Fisher Scientific) under the following reaction conditions: Holding stage, $95^{\circ} \mathrm{C}$ for $1 \mathrm{~min}$; cycling stage, $95^{\circ} \mathrm{C}$ for $15 \mathrm{sec}, 60^{\circ} \mathrm{C}$ for $1 \mathrm{~min}, 72^{\circ} \mathrm{C}$ for $30 \mathrm{sec}, 40$ cycles; melt curve stage, $95^{\circ} \mathrm{C}$ for $15 \mathrm{sec}, 55^{\circ} \mathrm{C}$ for $1 \mathrm{~min}, 95^{\circ} \mathrm{C}$ for $15 \mathrm{sec}$. Data analysis of the amplification results was performed using ABI's StepOne Software v2.2. Quantification was performed using the $2^{-\Delta \Delta \mathrm{Cq}}$ method (33).

Western blot analysis was performed as follows: Primary antibodies against the following proteins were used in this study: KRAS and $\beta$-actin. The antibodies against KRAS (ab180772, dilution: $1 / 200$ ) and $\beta$-actin (ab8227, dilution: 1/1,000) were purchased from Abcam Biotechnology Inc., and the other antibodies were purchased from Cell Signaling Technology). The cells were digested with $0.25 \%$ trypsin at $48 \mathrm{~h}$ prior to transfection. RIPA lysate (pre-added protease and phosphatase inhibitor) was added to the tube, and lysed on ice for $1 \mathrm{~h}$, during which the EP tube was inverted every $10 \mathrm{~min}$ to promote cell lysis. This was followed by centrifugation for $30 \mathrm{~min}$ at $4^{\circ} \mathrm{C}, 14,000 \mathrm{x}$. The supernatant was then carefully pipetted into a new EP tube, and a small amount was dispensed for protein concentration determination. The protein concentration was determined using the BCA Protein Assay kit. The protein concentration standard curve was prepared based on the protein standard concentration and the absorbance value. The protein concentration of the sample was calculated based on the protein concentration standard curve and the sample dilution factor. A suitable concentration of separation gel was prepared and added to the glass interlayer immediately after mixing. Deionized water was added to the upper layer of the glue and polymerized at room temperature for $30 \mathrm{~min}$. The ionized water was removed after condensation. The concentrated glue liquid was then added to the glass interlayer. The Teflon comb was inserted into the laminated glue liquid, and polymerization was carried out at room temperature for $30 \mathrm{~min}$. The protein sample was mixed with the loading buffer 
A

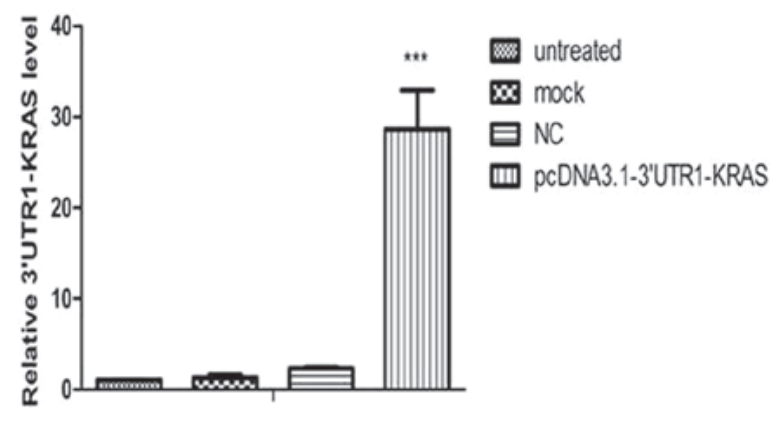

$\mathrm{C}$

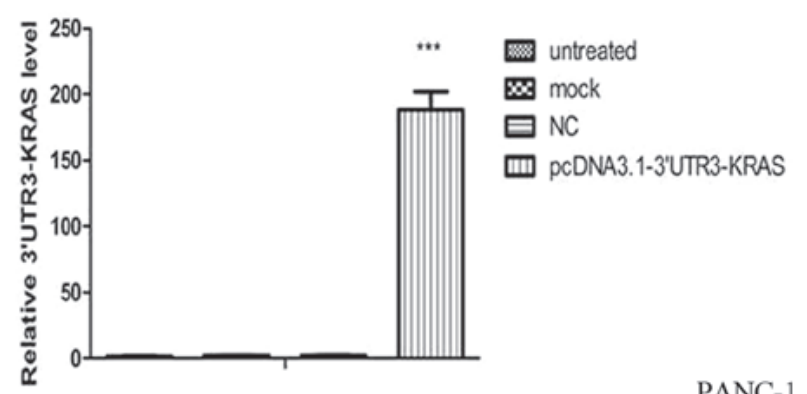

B

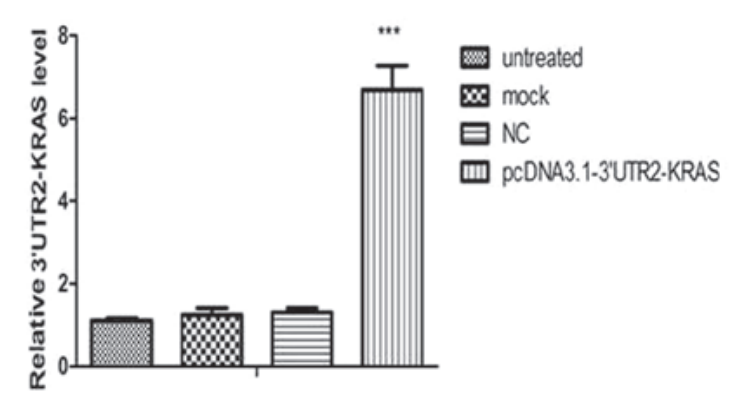

$\mathrm{D}$

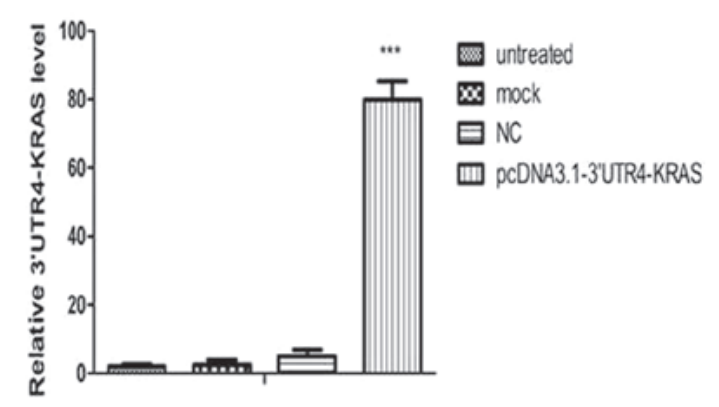

Figure 2. Construction of pcDNA3.1-KRAS-12xMS2bs. Compared with the untreated, mock and negative control (NC) groups, the (A) pcDNA3.1-3'UTR1KRAS-12xMS2bs, (B) pcDNA3.1-3'-UTR2-KRAS-12xMS2bs, (C) pcDNA3.1-3'-UTR3-KRAS-12xMS2bs and (D) pcDNA3.1-3'-UTR4-KRAS-12xMS2bs plasmid groups successfully expressed their corresponding $3^{\prime}-$-UTR-KRAS-12xMS2bs RNA (**** $\left.\mathrm{P}<0.001\right)$.

and incubated at $95^{\circ} \mathrm{C}$ for $5 \mathrm{~min}$. The protein sample was aspirated and electrophoresis was performed at a loading of 50-100 $\mu \mathrm{g} /$ well. The PVDF membrane was cut to the appropriate size and the upper left corner was cut as a marker. The PVDF membrane was equilibrated in methanol for $5 \mathrm{~min}$, and then equilibrated in $1 \mathrm{X}$ transfection buffer. The current intensity and film transfer time were adjusted according to the molecular weight of the protein, the thickness of the gel and the pore size of the PVDF membrane. The parameter settings were: $200 \mathrm{~mA}$ ice transfer for $2 \mathrm{~h}$; or $80 \mathrm{~mA}, 4^{\circ} \mathrm{C}$, transfer overnight. The transfer PVDF membrane was then removed, and we observed whether the pre-dyed Marker was completely transferred from the gel to the membrane; the PVDF membrane was then placed in 5\% TBST milk at room temperature for $1 \mathrm{~h}$. The corresponding antibody was diluted in the primary antibody dilution at an appropriate ratio, the PVDF membrane was removed from the milk, placed in the diluted primary antibody solution, and incubated overnight at $4^{\circ} \mathrm{C}$ with slow shaking. The PVDF membrane was removed from the primary antibody and washed 5 times with $1 \mathrm{X}$ TBST for $5 \mathrm{~min}$ each. The secondary antibody was diluted to a suitable titer with 5\% TBST milk, and the PVDF membrane was placed in a secondary antibody for $1 \mathrm{~h}$ at room temperature on a shaker. The PVDF membrane was removed from the secondary antibody and washed 4 times with $1 \mathrm{X}$ TBST for $5 \mathrm{~min}$ each time and $1 \mathrm{X}$ TBS for $5 \mathrm{~min}$. Subsequently, $1 \mathrm{ml}$ of each of the fluorescent luminescent liquids A and B was mixed (ECL kit, Cat. no. 32106; Pierce Biotechnology/Thermo Fisher Scientific), dropped on a PVDF membrane, and incubated for $\sim 1$ min. The luminescent liquid on the surface of the PVDF film was blotted. The PVDF film was placed in a cassette, and the
$\mathrm{X}$ film was completed in a dark room. The Thermo Scientific Pierce ECL Western Blotting Substrate is a highly sensitive non-radioactive, enhanced luminol-based chemiluminescent substrate for the detection of horseradish peroxidase (HRP) on immunoblots. Pierce ECL Western Blotting Substrate enables the detection of picogram amounts of antigen and allows for easy detection of HRP using photographic or other imaging methods. Blots can be repeatedly exposed to X-ray film to obtain optimal results. The western blots were analyzed using Quantity One software (Bio-Rad).

Statistical analysis. Statistical analyses were performed using SPSS 17.0 software (SPSS, Chicago, IL, USA). Multigroup comparisons of the means were carried out by one-way analysis of variance (ANOVA) with post hoc contrasts by the Student-Newman-Keuls test. Results are presented as the means \pm standard deviation. P-values $<0.05$ were considered to indicate statistically significant differences.

\section{Results}

Construction of pcDNA3.1-KRAS-12xMS2bs expression vector and KRAS-12xMS2bs RNA expression. Following the successful construction of the pcDNA3.1-KRAS-12xMS2bs plasmid (verified by Sanger sequencing), it was transfected into the PANC-1 cells using Lipofectamine 2000 reagent, and KRAS-12xMS2bs RNA expression was observed (Fig. 2). Compared with the untreated, mock and negative control (NC) groups, the pcDNA3.1-3'UTR1-KRAS-12xMS2bs (Fig. 2A), pcDNA3.1-3'-UTR2-KRAS-12xMS2bs (Fig. 2B), pcDNA3.1-3'-UTR3-KRAS-12xMS2bs (Fig. 2C) and 
Figure 3. Following isopropyl- $\beta$-D-1-thiogalactopyranoside (IPTG) induction, we observed the protein expression of supernatants and precipitation at different temperatures $\left(15,25\right.$ and $\left.37^{\circ} \mathrm{C}\right)$. The MBP-MS2 fusion protein was successfully expressed and weighed $\sim 60 \mathrm{kDa}$.

Table I. Small RNA fragment expression levels, sequences and precursors.

\begin{tabular}{lll}
\hline Expression level & \multicolumn{1}{c}{ Sequence } & \multicolumn{1}{c}{ Precursors } \\
\hline 686268 & 5'-TCCGCCATGTTGTTGGTGG-3' & hsa-let-7f \\
388787 & 5'-TGAGGTAGTAGGTTGTATAGTT-3' & hsa-let-7a \\
279651 & 5'-AACATTCAACGCTGTCGGTGAGT-3' & hsa-mir-181a \\
176094 & 5'-AAGCTGCCAGTTGAAGAACTGT-3' & hsa-mir-22 \\
121080 & 5'-TGTAAACATCCTCGACTGGAAGCT-3' & hsa-mir-30a \\
95592 & 5'-TTCACAGTGGCTAAGTTCTGC-3' & hsa-mir-27b \\
87762 & 5'-TGAGGTAGTAGTTTGTGCTGTT-3' & hsa-let-7i \\
85590 & 5'-AGCTACATCTGGCTACTGGGTCTC-3' & hsa-mir-222 \\
60084 & 5'-TGTAAACATCCCCGACTGGAAGCT-3' & hsa-mir-30d \\
58675 & 5'-TGTAAACATCCTTGACTGGAAGCT-3' & hsa-mir-30e \\
94729 & 5'-TGAGGTAGTAGTTTGTACAGTT-3' & hsa-let-7g \\
7859 & 5'-AGAGGTAGTAGGTTGCATAGTT-3' & hsa-let-7d \\
6654 & 5'-AACATTCAACGCTGTCGGTGA-3' & hsa-mir-181a \\
2353 & 5'-TGTAAACATCCTACACTCAGCT-3' & hsa-mir-30b \\
\hline
\end{tabular}

pcDNA3.1-3'-UTR4-KRAS-12xMS2bs (Fig. 2D) plasmid groups successfully expressed their corresponding 3'-UTR-KRAS12xMS2bs RNA $(\mathrm{P}<0.001)$. At the same time, the successful production of the MBP-MS2 fusion protein was observed (Fig. 3).

miRNA sequencing. Base composition and quality value distribution maps of the filtered data indicated the samples with balanced base compositions. Each point represents the base quality value and corresponding position of each read (Fig. 4A). The base mass distribution of reads. The abscissa is the position of the base distributed over the read, and the ordinate represents the mass of the base. Each point in the graph represents the base quality value of the corresponding position in a read. Overall, if the low-mass $(<20)$ base ratio is low, the sequencing quality of this lane is better (Fig. 4B).

The distribution of small RNAs among different categories [such as small cytoplasmic RNAs (scRNAs), snRNAs, rRNAs and miRNAs] is shown in Fig. 5. The data on the expression levels of small RNA fragments, their sequences and precursors are presented in Table I. Family analysis revealed that 
Base percentage composition along reads

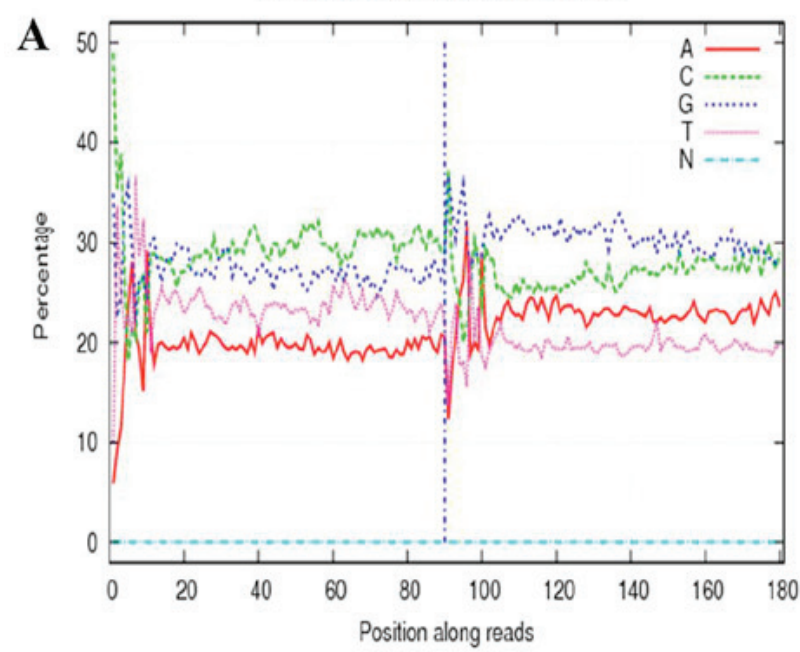

Distribution of qualifies

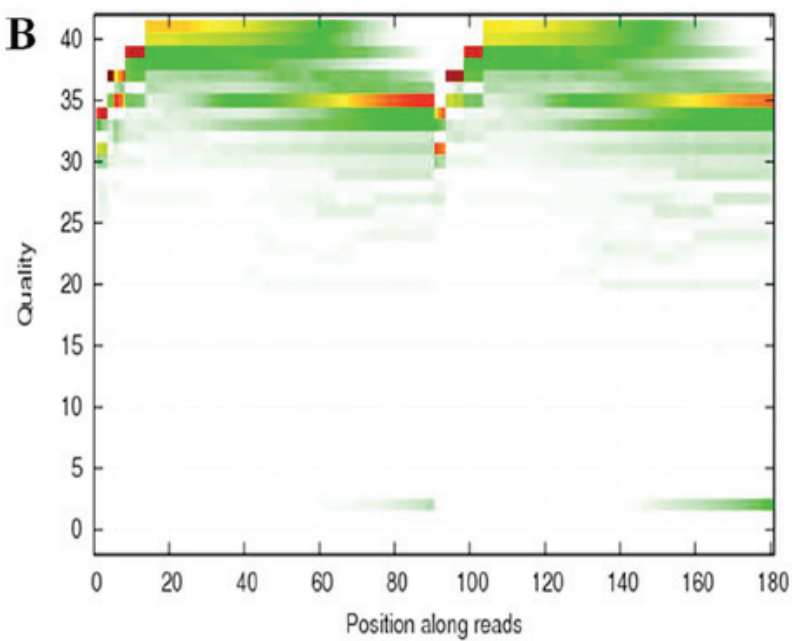

Figure 4. Base composition and quality distribution along sequencing reads. (A) On the x-axis, position 1-90 bp represents read 1 and $91-180$ bp represents read 2. The A-curve should overlap with the T-curve, while the G-curve overlapped with the C-curve. The case presented shows a balanced composition (B) The $\mathrm{x}$-axis represents positions along reads and the $y$-axis is quality value. If the percentage of bases with low quality $(<20)$ is low, the sequencing quality of this lane is considered satisfactory.

\title{
Pie chart for annotation_KRAS-treated-uniq
}

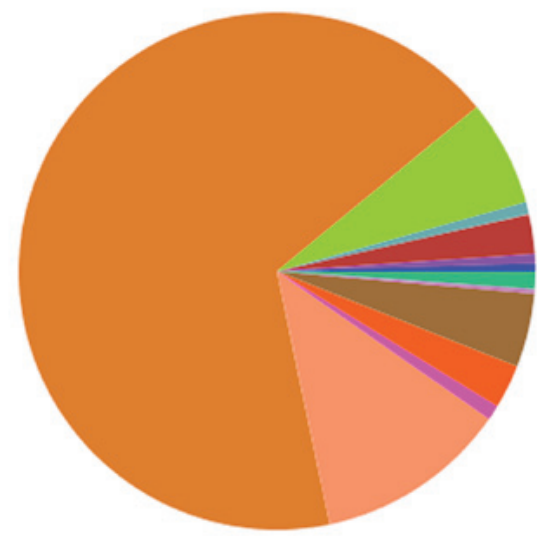

\author{
scRNA (2193) \\ intron_antisense (2753) \\ intron_sense (11558) \\ srpRNA (72) \\ snRNA (3665) \\ exon_sense (30793) \\ unann (314303) \\ rRNA (56253) \\ snoRNA (4225) \\ repeat $(12825)$ \\ tRNA (21630) \\ exon_antisense (1229) \\ miRNA (5184)
}

Figure 5. Distribution of small RNAs among different categories following RNA sequencing [such as small cytoplasmic RNAs (scRNAs), small nuclear RNAs (snRNAs), ribosomal RNAs (rRNAs) and microRNAs (miRNAs)]. Small RNAs (sRNAs) from HiSeq deep sequencing cover almost every kind of RNA, including miRNA, siRNA, piRNA, rRNA, tRNA, snRNA, snoRNA, repeat associated sRNA and degraded tags of exon or intron, of which miRNA, siRNA and piRNA are hot topics in the research field of small RNA.

the identified miRNAs belonged to the let-7 and miR-34, -30 and -143 families (data not shown). KRAS has been shown to interact with miRNA from these families, thereby affecting cell proliferation, apoptosis and migration.

miRNA prediction data also included small RNAs that did not annotate to any database upon classification, but did annotate to the genomic, intronic and exonic antisense strand sequences. The 21 newly predicted miRNAs are listed in Table II. Precursor prediction resulted in 338 unique novel miRNA precursor candidates and 6,311 total miRNA precursor candidates. Comparison of newly predicted miRNAs to the miRBase database revealed that a proportion belonged to the miR-345, $-330,-574$ and -1243 families (data not shown).
lncRNA-related miRNA precursor prediction. Following Dicer and Drosha enzyme $(34,35)$ shearing, lncRNAs form miRNAs, which then exert their corresponding effects. Thus, we matched our IncRNAs to miRBase to identify potential miRNA precursors. IncRNAs with $>90 \%$ coverage were selected. MiRPara software (36) was also used to predict miRNA and their precursors (Table III). A total of 91 known miRNA precursors and 1,381 predicted new miRNA precursors were identified (data not shown).

lncRNA family prediction. IncRNA family class predictions were made based on structural and sequence features. Rfam is a database that contains information on various non-coding RNA families, including conserved regions of RNA secondary 
Table II. New microRNA precursor statistics in samples.

\begin{tabular}{ll}
\hline Name & \multicolumn{1}{c}{ Nucleotide sequence } \\
\hline t0004126 & 5'-AGGAACCTTGGAGCTTCGGCAGC-3' \\
t0016193 & 5'-TCTGACTCCTAGTCCAGGGCT-3' \\
t0019851 & 5'-ACCAGGCAAGAACTACTGTCT-3' \\
t0027353 & 5'-AGGAACCTTGGAGCTTCGGCAG-3' \\
t0031267 & 5'-CAAATGGATAGGATAACACCT-3' \\
t0037092 & 5'-TGACTGCAGCTACTCTCCCCAAG-3' \\
t0046425 & 5'-CAAAGCACACGGCCTGCAGAGT-3' \\
t0050628 & 5'-TGAGTGTGTGTGTGTGAGTGTGA-3' \\
t0057669 & 5'-TCGAGAATTGCGTTTGGACAAT-3' \\
t0065832 & 5'-TCACTACCTGACAATACAGCAGT-3' \\
t0067365 & 5'-CAGCCAGCAGGAGAGAGAGGGAGC-3' \\
t0068481 & 5'-ATTCCTGTAACTGGGCCAGTTT-3' \\
t0068961 & 5'-TTCCTGTGATGTTCCTGAGGAAG-3' \\
t0078411 & 5'-AGGAACCTTGGAGCTTCGGCA-3' \\
t0084668 & 5'-GGAGGAACCTTGGAGCTTCGGCA-3' \\
t0137108 & 5'-CGCGGGGCTCCGCGGGCGGCGA-3' \\
t0140064 & 5'-CGCGGGGCTCCGCGGGCGGCGAG-3' \\
t0160363 & 5'-TCTGACTCCTAGTCCAGGGC-3' \\
t0240262 & 5'-CACGATGATGACACTGAGG-3' \\
t0299819 & 5'-TGAGTGTGTGTGTGTGAGTGTGAAC-3' \\
t0421760 & 5'-CGGGGAGAGGTCTGGGGAA-3' \\
\hline
\end{tabular}

Table III. Long non-coding RNA (lncRNA) names and their corresponding small RNA precursors.

\begin{tabular}{ll}
\hline lncRNA & Corresponding small RNA precursor \\
\hline TCONS_00062686 & hsa-let-7a-1 \\
n407573 & hsa-let-7a-3 \\
n407573 & hsa-let-7b \\
TCONS_00062686 & hsa-let-7f-1 \\
TCONS_00063039 & hsa-mir-181a-2 \\
TCONS_00014041 & hsa-let-7i \\
n386735 & hsa-mir-18a \\
n386735 & hsa-mir-20a \\
n408292 & hsa-mir-22 \\
TCONS_00019276 & hsa-mir-134 \\
TCONS_00019278 & hsa-mir-154 \\
TCONS_00019274 & hsa-mir-300 \\
\hline
\end{tabular}

structures, cis-acting RNA elements, as well as other elements. The newly predicted IncRNA families included MALAT1, MEG3_2, TUG1_1-4, KCNQ1OT1_2, NEAT1_3, ZEB2_ AS1_3 and DLEU1_1 (Table IV).

Function verification. miRNA mimics of highly expressed small fragments matching several miRNA precursors (hsa-let-7a, hsa-let-7i and hsa-miR-30b) were synthesized (Shanghai GenePharma Co., Ltd.) and transfected into PC cells. The results demonstrated a decreased KRAS mRNA
Table IV. Long non-coding RNA (lncRNA) family analysis.

\begin{tabular}{ll}
\hline Family name & IncRNA \\
\hline MALAT1 & TCONS_00012377 \\
MEG3_2 & TCONS_00019252 \\
TUG1_1 & TCONS_00040841 \\
TUG1_2 & TCONS_00040841 \\
TUG1_3 & TCONS_00040841 \\
TUG1_4 & TCONS_00040841 \\
KCNQ1OT1_2 & TCONS_00037627 \\
NEAT1_3 & TCONS_00012376 \\
ZEB2_AS1_3 & TCONS_00022706 \\
DLEU1_1 & TCONS_00016977 \\
DLEU1_2 & TCONS_00017846 \\
DLEU2_1 & TCONS_00017847 \\
DLEU2_2 & TCONS_00017851 \\
DLEU2_3 & TCONS_00017854 \\
DLEU2_6 & TCONS_00017854 \\
FAM13A & TCONS_00037223 \\
FTX_1 & TCONS_00066659 \\
JPX_1 & TCONS_00065421 \\
NBR2 & TCONS_00025667 \\
\hline
\end{tabular}

and protein expression in the transfected cells (Fig. 6). Compared with the untreated and negative control (NC) groups, KRAS mRNA (Fig. 6A) and protein (Fig. 6B) levels were both decreased in the hsa-let-7a, hsa-let-7i and hsa-miR$30 \mathrm{~b}$ groups.

\section{Discussion}

PC is difficult to detect in its early stages, and metastasis often occurs long before patients develop clinical symptoms, making surgical resection more difficult. Furthermore, PC is highly aggressive and resistant to the majority of chemotherapeutic agents, resulting in a high mortality rate. KRAS, a member of the $R A S$ gene family, is widely known to be closely associated with PC. KRAS mutations are the most common in malignant human cancers (37). Point mutations frequently occur at codon 12 (GGT to GAT, GTT, or CGT) in $\mathrm{PC}$ cells. The mutation detection rate is $<30 \%$ in early stages of tumorigenesis, increasing to $\sim 100 \%$ in late-stage PC (38). Generally, KRAS mutations lead to the abnormal activation of cell proliferation and survival pathways, thereby playing a key role in PC development.

miR-126 and miR-143 have been shown to act as tumor suppressors by targeting KRAS $(39,40)$, which may prove beneficial for patients with PC. Compared with conventional chemotherapeutic drugs, miRNA molecules are able to target a certain gene or genes, which would eliminate the occurrence of serious adverse reactions or side-effects caused by radiotherapy and chemotherapy. Compared with RNA interference technology, miRNAs are endogenous small molecules that do not elicit allergic or other rejection reactions in the body. During the process of tumor development, some miRNAs are found at decreased levels or even disappear. If their levels are increased 
A

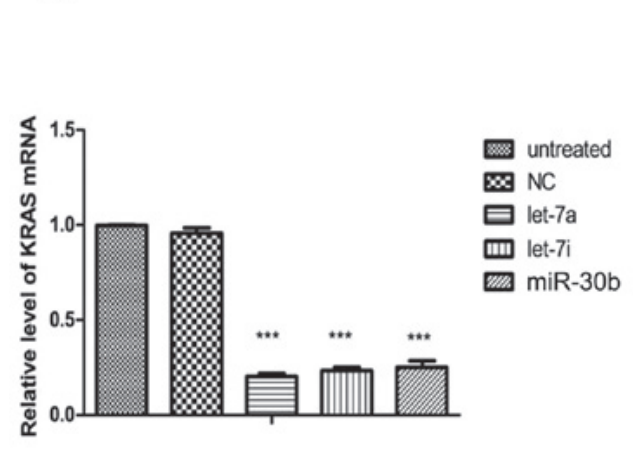

B

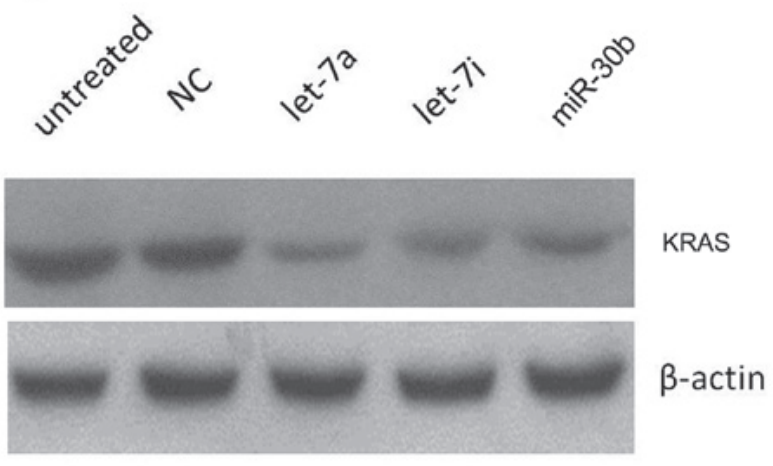

Figure 6. KRAS expression levels following transfection of small RNA fragment mimics. Compared with the untreated and negative control (NC) groups, $K R A S(\mathrm{~A})$ mRNA and (B) protein levels were both reduced in the hsa-let-7a, hsa-let-7i and hsa-miR-30b groups.

or restored to normal, it is possible that the malignant behavior of tumors could be restrained or eliminated. Therefore, gene therapy strategies involving miRNAs for the treatment of aggressive diseases, such as PC, should be established. Using miRNAs to inhibit target genes has many potential advantages and is expected to become a new method for the study of gene function and a therapy with broad applications. In the present study, we investigated the use of miRNAs to inhibit the KRAS oncogene in order to develop an effective gene therapy for PC.

Overall, we sequenced high levels of several miRNAs known to target KRAS, such as let-7, miR-30 and miR-34, demonstrating that our method of specific MS2-MBP and KRAS-MS2bs affinity chromatography screening of associated non-coding RNAs is quite feasible. In the present study, the highly expressed small fragments were found to have miRNA precursors such as hsa-let-7f, -7i, -7a and -7g, and hsamiR-30a, -30e, -27a, -27b, -222, -10a, -10b, -181a and -96. The analysis of known miRNA families revealed that they belong to the let-7 and miR-34, -30 and -143 families. The transfection of synthetic miRNA mimics of selected highly expressed small fragments into PC cells led to a decrease in KRAS protein levels. Johnson et al first observed that the expression level of let-7 in lung cancer was decreased, while $R A S$ expression was increased, confirming the target relationship between the two (41). Kumar et al found that the elevated let-7 expression was able to significantly inhibit the growth of lung cancer cells in humans and mice (42). Let-7 was also found to be downregulated in a number of tumors, such as breast, colon, ovarian and prostate cancers. The loss of let-7 expression in these tumors plays an important role in promoting the tumorigenic process (43). Torrisani et al confirmed that KRAS inhibition by let-7 was weaker in the pathogenesis of PC (44). Kent et al confirmed that miR-143/145 was able to inhibit the expression of $K R A S$ and downstream RREBl (45). In addition, the effect of miRNA on KRAS has also been reported in other tumors. Tsang et al confirmed that miR-18a acts as a tumor suppressor by inhibiting KRAS in squamous cell carcinoma, colon cancer and embryonic liver cells (46), whereas Shin et al found that miR-181 downregulated KRAS in oral squamous cell carcinoma (47). However, the role of miR-18a and miR-181 in PC remains to be confirmed by further studies. Several miRNA molecules (miR-193b, -217 and -96) are downregulated in PC. These miRNAs inhibit PC cell proliferation, invasion and metastasis by targeting $\operatorname{KRAS}(7,10,11)$. Based on our current understanding of miRNAs, many more potential miRNAs remain to be further investigated.

Studies have demonstrated that hsa-let-7a targets KRAS through its 3'-UTR (48). hsa-let-7g is associated with liver and breast cancer and tumor metastasis, and regulates cell proliferation and migration through interacting with KRAS (49). It has also been demonstrated that miR-27a acts on the 3 '-UTR of $K R A S$ to inhibit esophageal protein expression (50). Similarly, the present study detected high expression levels of hsa-miR-27a. In colorectal cancer, miR-30b and miR-143 target $K R A S$, acting as tumor suppressors $(51,52)$, whereas miR-30c was shown to regulate $K R A S$ expression by combining with its 3'-UTR in breast cancer (53). Our previous study demonstrated that miR-96 also targets KRAS (11). As research time was limited, the experiment of verification of KRAS-3'UTR enrichment and the effective enrichment of the targets in other cell lines will be performed in a future study.

In the present study, we also predicted a total of 21 new miRNAs associated with PC, but their expression levels were low. We also completed secondary structure diagrams of 338 new pre-miRNAs. The comparison of predicted miRNAs to the miRBase database revealed they belong to the miR-345, $-330,-574$ and -1243 families. However, after transfecting synthetic miRNA mimics of the predicted miRNAs into PC cells, KRAS protein expression was not significantly affected. A possible explanation may be that these miRNAs may play an indirect role or act synergistically with other miRNAs or non-coding RNAs; in this case, KRAS regulation may not be readily obvious when they are individually transfected into PC cells.

The role of non-coding lncRNAs in the development of tumors has become a focus of investigation. IncRNA HEIH is highly expressed in hepatocellular carcinoma and is an important proto-oncogene that promotes the progression of this type of cancer (54). IncRNA PCAT-1 is PC-specific and known to inhibit BRCA2 expression. It promotes cancer cell proliferation in vitro and in vivo and plays an important role in the progression of PC $(55,56)$. Moreover, Gas 5 was found to be downregulated in breast cancer cells. Gas 5 overexpression can induce apoptosis in breast cancer cells and inhibit their proliferation (57). IncRNA $M E G 3$ is a maternally imprinted gene found to downregulate human gastric, cervical and 
non-small-cell lung cancer, among others. MEG3 transfection into HeLa, A549 and other human tumor cell lines can inhibit tumor cell proliferation and has been confirmed to act as a tumor suppressor by activation of p53 signaling $(58,59)$.

Unlike protein-coding genes, the role of lncRNAs in PC carcinogenesis and development remains unclear. As there is a higher level of temporal, spatial and tissue-specific lncRNA expression, there is great potential in predicting new lncRNA involvement in cancers and that may provide valuable downstream functional data. MALAT1 has been shown to be highly expressed in PC. MALAT1 expression is significantly associated with tumor size, stage and depth of invasion, and patients with high expression of MALAT1 have a worse prognosis (60). Lu et al, as well as others, reported that the IncRNA Gas 5 negatively regulates the expression of CDK6, inducing proliferation of PC cells (61). Kim et al demonstrated that IncRNA HOTAIR acts as an oncogene in PC (20). Sun et $a l$, as well as others, observed low expression of a new lncRNA, ENST00000480739, in PC; this lncRNA targets HIF-1 $\alpha$ to increase OS-9 expression (62). It has been demonstrated that the IncRNA H19 increased HMGA2-mediated epithelial-to-mesenchymal transition through inhibition of let-7, thereby promoting the invasion and metastasis of $\mathrm{PC}$ cells (63). However, the role of lncRNAs in PC has not been extensively investigated to date. Dicer and Drosha enzyme shearing of lncRNAs results in the formation of miRNAs, which can then exert their corresponding cellular effects. In our experiments, we found the corresponding small precursor of IncRNA TCONS_00062686 was hsa-let-7a-1, that of $n 407573$ was hsa-let-7a-3, the precursor of TCONS_00062686 was hsa-let-7f-1, the precursor of TCONS_00063039 was hsa-miR-181a-2, and the precursor of $n 386735$ was hsa-miR-18a. All these lncRNAs and their miRNA precursors play a key role in tumorigenesis by interacting with KRAS. In the present study, we obtained the miRNA and lncRNA data of interest through next-generation RNA sequencing. We identified several miRNA precursors that belong to the let-7 and miR-34, -30 and -143 families, as well as relevant lncRNAs and their families (MALAT1, MEG3_2 and TUG1_1-4).

In future experiments, our aims are to synthesize and transfect the corresponding small interfering RNA resulting from lncRNA cleavage into PC cells, and KRAS protein and mRNA levels to be monitored, along with an extensive analysis of the mutual regulation between the obtained lncRNA and miRNA. Further verification of the obtained lncRNA expression in PC paraffin-embedded tissues will be conducted through RNAscope in situ hybridization in future experiments, along with an analysis of miRNA and lncRNA expression levels in blood samples from PC patient. Our databank of non-coding RNAs (mainly miRNAs and lncRNAs) that regulate $K R A S$ will greatly improve our understanding of KRAS regulation-associated tumorigenesis and hopefully lead to the design of gene therapies for PC. The results should reveal a complex regulatory network that may provide new insight and novel approaches for more effective PC treatment strategies.

\section{Acknowledgements}

Not applicable.

\section{Funding}

This study was supported by the National Nature Science Foundations of China (grant nos. 8172334, 81341070 and 81472326).

\section{Availability of data and materials}

The datasets used and/or analyzed during the current study are available from the corresponding author on reasonable request.

\section{Authors' contributions}

LZ was involved in the conception and design of the study, acquisition of data, analysis and interpretation of the data, and in the drafting of the article; SY, CW and CJ were involved in the acquisition of data; JC and ZL were involved in the conception and design of the study; JC gave the final approval of the article. All authors have reviewed and approved the final version of the manuscript.

\section{Ethics approval and consent to participate}

Not applicable.

\section{Patient consent for publication}

Not applicable.

\section{Competing interests}

The authors declare that they have no competing interests to disclose.

\section{References}

1. Ilic M and Ilic I: Epidemiology of pancreatic cancer. World J Gastroenterol 22: 9694-9705, 2016.

2. Morris JP IV, Wang SC and Hebrok M: KRAS, Hedgehog, Wnt and the twisted developmental biology of pancreatic ductal adenocarcinoma. Nat Rev Cancer 10: 683-695, 2010.

3. Ying H, Dey P, Yao W, Kimmelman AC, Draetta GF, Maitra A and DePinho RA: Genetics and biology of pancreatic ductal adenocarcinoma. Genes Dev 30: 355-385, 2016.

4. Bartel DP: MicroRNAs: Genomics, biogenesis, mechanism, and function. Cell 116: 281-297, 2004.

5. Bloomston M, Frankel WL, Petrocca F, Volinia S, Alder H, Hagan JP, Liu CG, Bhatt D, Taccioli C and Croce CM: MicroRNA expression patterns to differentiate pancreatic adenocarcinoma from normal pancreas and chronic pancreatitis. JAMA 297: 1901-1908, 2007.

6. Rachagani S, Macha MA, Menning MS, Dey P, Pai P, Smith LM, Mo YY and Batra SK: Changes in microRNA (miRNA) expression during pancreatic cancer development and progression in a genetically engineered KrasG12D;Pdx1-Cre mouse (KC) model. Oncotarget 6: 40295-40309, 2015.

7. Jin X, Sun Y, Yang H, Li J, Yu S, Chang X, Lu Z and Chen J: Deregulation of the miR-193b-KRAS axis contributes to impaired cell growth in pancreatic cancer. PLoS One 10: e0125515, 2015.

8. Keklikoglou I, Hosaka K, Bender C, Bott A, Koerner C, Mitra D, Will R, Woerner A, Muenstermann E, Wilhelm H, et al: MicroRNA-206 functions as a pleiotropic modulator of cell proliferation, invasion and lymphangiogenesis in pancreatic adenocarcinoma by targeting ANXA2 and KRAS genes. Oncogene 34: 4867-4878, 2015.

9. Hu Y, Ou Y, Wu K, Chen Y and Sun W: miR-143 inhibits the metastasis of pancreatic cancer and an associated signaling pathway. Tumour Biol 33: 1863-1870, 2012. 
10. Zhao WG, Yu SN, Lu ZH, Ma YH, Gu YM and Chen J: The miR-217 microRNA functions as a potential tumor suppressor in pancreatic ductal adenocarcinoma by targeting KRAS Carcinogenesis 31: 1726-1733, 2010

11. Yu S, Lu Z, Liu C, Meng Y, Ma Y, Zhao W, Liu J, Yu J and Chen J: miRNA-96 suppresses KRAS and functions as a tumor suppressor gene in pancreatic cancer. Cancer Res 70: 6015-6025, 2010

12. Wapinski $\mathrm{O}$ and Chang HY: Long noncoding RNAs and human disease. Trends Cell Biol 21: 354-361, 2011.

13. Novikova IV, Hennelly SP, Tung CS and Sanbonmatsu KY: Rise of the RNA machines: Exploring the structure of long non-coding RNAs. J Mol Biol 425: 3731-3746, 2013.

14. Tripathi V, Ellis JD, Shen Z, Song DY, Pan Q, Watt AT, Freier SM, Bennett CF, Sharma A, Bubulya PA, et al: The nuclear-retained noncoding RNA MALAT1 regulates alternative splicing by modulating SR splicing factor phosphorylation. Mol Cell 39: 925-938, 2010.

15. Wang KC, Yang YW, Liu B, Sanyal A, Corces-Zimmerman R, Chen Y, Lajoie BR, Protacio A, Flynn RA, Gupta RA, et al: A long noncoding RNA maintains active chromatin to coordinate homeotic gene expression. Nature 472: 120-124, 2011.

16. Muers M: RNA: Genome-wide views of long non-coding RNAs. Nat Rev Genet 12: 742-743, 2011

17. Chen X and Yan GY: Novel human lncRNA-disease association inference based on lncRNA expression profiles. Bioinformatics 29: 2617-2624, 2013

18. Harries LW: Long non-coding RNAs and human disease. Biochem Soc Trans 40: 902-906, 2012.

19. Tahira AC, Kubrusly MS, Faria MF, Dazzani B, Fonseca RS Maracaja-Coutinho V, Verjovski-Almeida S, Machado MC and Reis EM: Long noncoding intronic RNAs are differentially expressed in primary and metastatic pancreatic cancer. Mol Cancer 10: 141, 2011.

20. Kim K, Jutooru I, Chadalapaka G, Johnson G, Frank J, Burghardt R, Kim S and Safe S: HOTAIR is a negative prognostic factor and exhibits pro-oncogenic activity in pancreatic cancer. Oncogene 32: 1616-1625, 2013.

21. Li Z, Zhao X, Zhou Y, Liu Y, Zhou Q, Ye H, Wang Y, Zeng J, Song Y, Gao W, et al: The long non-coding RNA HOTTIP promotes progression and gemcitabine resistance by regulating HOXA13 in pancreatic cancer. J Transl Med 13: 84, 2015.

22. Pang EJ, Yang R, Fu XB and Liu YF: Overexpression of long non-coding RNA MALAT1 is correlated with clinical progression and unfavorable prognosis in pancreatic cancer. Tumour Biol 36: 2403-2407, 2015.

23. Peng W, Gao W and Feng J: Long noncoding RNA HULC is a novel biomarker of poor prognosis in patients with pancreatic cancer. Med Oncol 31: 346, 2014.

24. Liu JH, Chen G, Dang YW, Li CJ and Luo DZ: Expression and prognostic significance of lncRNA MALAT1 in pancreatic cancer tissues. Asian Pac J Cancer Prev 15: 2971-2977, 2014.

25. Kuzmanovic DA, Elashvili I, Wick C, O'Connell C and Krueger S: The MS2 coat protein shell is likely assembled under tension: A novel role for the MS2 bacteriophage A protein as revealed by small-angle neutron scattering. J Mol Biol 355: 1095-1111, 2006.

26. Batey RT and Kieft JS: Improved native affinity purification of RNA. RNA 13: 1384-1389, 2007.

27. Gong C, Popp MW and Maquat LE: Biochemical analysis of long non-coding RNA-containing ribonucleoprotein complexes. Methods 58: 88-93, 2012

28. Zhou Z and Reed R: Purification of functional RNA-protein complexes using MS2-MBP. Curr Protoc Mol Biol Chapter 27: $3,2003$.

29. Corcoran CP, Rieder R, Podkaminski D, Hofmann B and Vogel J: Use of aptamer tagging to identify in vivo protein binding partners of small regulatory RNAs. Methods Mol Bio 905: 177-200, 2012.

30. Said N, Rieder R, Hurwitz R, Deckert J, Urlaub H and Vogel J: In vivo expression and purification of aptamer-tagged smal RNA regulators. Nucleic Acids Res 37: e133, 2009.

31. Bardwell VJ and Wickens M: Purification of RNA and RNA-protein complexes by an R17 coat protein affinity method. Nucleic Acids Res 18: 6587-6594, 1990

32. SenGupta DJ, Zhang B, Kraemer B, Pochart P, Fields S and Wickens M: A three-hybrid system to detect RNA-protein interactions in vivo. Proc Natl Acad Sci USA 93: 8496-8501, 1996.

33. Livak KJ and Schmittgen TD: Analysis of relative gene expression data using real-time quantitative PCR and the 2(- $\Delta \Delta \mathrm{C}(\mathrm{T}))$ Method. Methods 25: 402-408, 2001.
34. Cai X, Hagedorn $\mathrm{CH}$ and Cullen BR: Human microR NAs are processed from capped, polyadenylated transcripts that can also function as mRNAs. RNA 10: 1957-1966, 2004.

35. Lee Y, Kim M, Han J, Yeom KH, Lee S, Baek SH and Kim VN: MicroRNA genes are transcribed by RNA polymerase II. EMBO J 23: 4051-4060, 2004

36. Wu Y, Wei B, Liu H, Li T and Rayner S: MiRPara: A SVM-based software tool for prediction of most probable microRNA coding regions in genome scale sequences. BMC Bioinformatics 12: 107, 2011.

37. Pylayeva-Gupta Y, Grabocka E and Bar-Sagi D: RAS oncogenes: Weaving a tumorigenic web. Nat Rev Cancer 11: 761-774, 2011.

38. Rozenblum E, Schutte M, Goggins M, Hahn SA, Panzer S, Zahurak M, Goodman SN, Sohn TA, Hruban RH, Yeo CJ, et al: Tumor-suppressive pathways in pancreatic carcinoma. Cancer Res 57: 1731-1734, 1997.

39. Jiao LR, Frampton AE, Jacob J, Pellegrino L, Krell J, Giamas G, Tsim N, Vlavianos P, Cohen P, Ahmad R, et al: MicroRNAs targeting oncogenes are down-regulated in pancreatic malignant transformation from benign tumors. PLoS One 7: e32068, 2012.

40. Xu B, Niu X, Zhang X, Tao J, Wu D, Wang Z, Li P, Zhang W, Wu H, Feng N, et al: miR-143 decreases prostate cancer cells proliferation and migration and enhances their sensitivity to docetaxel through suppression of KRAS. Mol Cell Biochem 350: 207-213, 2011

41. Johnson SM, Grosshans H, Shingara J, Byrom M, Jarvis R, Cheng A, Labourier E, Reinert KL, Brown D and Slack FJ: RAS is regulated by the let-7 microRNA family. Cell 120: 635-647, 2005.

42. Kumar MS, Erkeland SJ, Pester RE, Chen CY, Ebert MS, Sharp PA and Jacks T: Suppression of non-small cell lung tumor development by the let-7 microRNA family. Proc Natl Acad Sci USA 105: 3903-3908, 2008.

43. Boyerinas B, Park SM, Hau A, Murmann AE and Peter ME: The role of let-7 in cell differentiation and cancer. Endocr Relat Cancer 17: F19-F36, 2010.

44. Torrisani J, Bournet B, du Rieu MC, Bouisson M, Souque A, Escourrou J, Buscail L and Cordelier P: let-7 MicroRNA transfer in pancreatic cancer-derived cells inhibits in vitro cell proliferation but fails to alter tumor progression. Hum Gene Ther 20: 831-844, 2009

45. Kent OA, Chivukula RR, Mullendore M, Wentzel EA, Feldmann G, Lee KH, Liu S, Leach SD, Maitra A and Mendell JT: Repression of the miR-143/145 cluster by oncogenic Ras initiates a tumor-promoting feed-forward pathway. Genes Dev 24: 2754-2759, 2010

46. Tsang WP and Kwok TT: The miR-18a* microRNA functions as a potential tumor suppressor by targeting on K-Ras. Carcinogenesis 30: 953-959, 2009.

47. Shin KH, Bae SD, Hong HS, Kim RH, Kang MK and Park NH: miR-181a shows tumor suppressive effect against oral squamous cell carcinoma cells by downregulating K-ras. Biochem Biophys Res Commun 404: 896-902, 2011.

48. Wang XR, Luo H, Li HL, Cao L, Wang XF, Yan W, Wang YY, Zhang JX, Jiang T, Kang CS, et al; Chinese Glioma Cooperative Group (CGCG): Overexpressed let-7a inhibits glioma cell malignancy by directly targeting K-ras, independently of PTEN. Neuro-oncol 15: 1491-1501, 2013.

49. Chen KJ, Hou Y, Wang K, Li J, Xia Y, Yang XY, Lv G, Xing XL and Shen F: Reexpression of Let-7g microRNA inhibits the proliferation and migration via K-Ras/HMGA2/snail axis in hepatocellular carcinoma. Biomed Res Int 2014: 742417, 2014.

50. Zhu L, Wang Z, Fan Q, Wang R and Sun Y: microRNA-27a functions as a tumor suppressor in esophageal squamous cell carcinoma by targeting KRAS. Oncol Rep 31: 280-286, 2014

51. Liao WT, Ye YP, Zhang NJ, Li TT, Wang SY, Cui YM, Qi L, Wu P, Jiao HL, Xie YJ, et al: MicroRNA-30b functions as a tumour suppressor in human colorectal cancer by targeting KRAS, PIK3CD and BCL2. J Pathol 232: 415-427, 2014.

52. Chen X, Guo X, Zhang H, Xiang Y, Chen J, Yin Y, Cai X, Wang K, Wang G, Ba Y, et al: Role of miR-143 targeting KRAS in colorectal tumorigenesis. Oncogene 28: 1385-1392, 2009.

53. Tanic M, Yanowsky K, Rodriguez-Antona C, Andrés R, Márquez-Rodas I, Osorio A, Benitez J and Martinez-Delgado B: Deregulated miRNAs in hereditary breast cancer revealed a role for miR-30c in regulating KRAS oncogene. PLoS One 7: e38847, 2012.

54. Yang F, Zhang L, Huo XS, Yuan JH, Xu D, Yuan SX, Zhu N, Zhou WP, Yang GS, Wang YZ, et al: Long noncoding RNA high expression in hepatocellular carcinoma facilitates tumor growth through enhancer of zeste homolog 2 in humans. Hepatology 54: $1679-1689,2011$ 
55. Prensner JR, Iyer MK, Balbin OA, Dhanasekaran SM, Cao Q, Brenner JC, Laxman B, Asangani IA, Grasso CS, Kominsky HD, et al: Transcriptome sequencing across a prostate cancer cohort identifies PCAT-1, an unannotated lincRNA implicated in disease progression. Nat Biotechnol 29: 742-749, 2011.

56. Prensner JR, Chen W, Iyer MK, Cao Q, Ma T, Han S, Sahu A, Malik R, Wilder-Romans K, Navone N, et al: PCAT-1, a long noncoding RNA, regulates BRCA2 and controls homologous recombination in cancer. Cancer Res 74: 1651-1660, 2014.

57. Mourtada-Maarabouni M, Pickard MR, Hedge VL, Farzaneh F and Williams GT: GAS5, a non-protein-coding RNA, controls apoptosis and is downregulated in breast cancer. Oncogene 28: 195-208, 2009.

58. Lu KH, Li W, Liu XH, Sun M, Zhang ML, Wu WQ, Xie WP and Hou YY: Long non-coding RNA MEG3 inhibits NSCLC cells proliferation and induces apoptosis by affecting p53 expression. BMC Cancer 13: 461, 2013.

59. Qin R, Chen Z, Ding Y, Hao J, Hu J and Guo F: Long non-coding RNA MEG3 inhibits the proliferation of cervical carcinoma cells through the induction of cell cycle arrest and apoptosis. Neoplasma 60: 486-492, 2013.
60. Jiao F, Hu H, Yuan C, Wang L, Jiang W, Jin Z, Guo Z and Wang L: Elevated expression level of long noncoding RNA MALAT-1 facilitates cell growth, migration and invasion in pancreatic cancer. Oncol Rep 32: 2485-2492, 2014.

61. Lu X, Fang Y, Wang Z, Xie J, Zhan Q, Deng X, Chen H, Jin J, Peng C, Li H, et al: Downregulation of gas 5 increases pancreatic cancer cell proliferation by regulating CDK6. Cell Tissue Res 354: 891-896, 2013.

62. Sun YW, Chen YF, Li J, Huo YM, Liu DJ, Hua R, Zhang JF, Liu W, Yang JY, Fu XL, et al: A novel long non-coding RNA ENST00000480739 suppresses tumour cell invasion by regulating OS-9 and HIF-1 $\alpha$ in pancreatic ductal adenocarcinoma. $\mathrm{Br}$ J Cancer 111: 2131-2141, 2014.

63. Ma C, Nong K, Zhu H, Wang W, Huang X, Yuan Z and Ai K: H19 promotes pancreatic cancer metastasis by derepressing let-7's suppression on its target HMGA2-mediated EMT. Tumour Biol 35: 9163-9169, 2014. 